\title{
Idiopathic granulomatous mastitis: factors influencing recovery and recurrence
}

\author{
Hüseyin Onur Aydın๑, Ataç Baykal๑, Ali Konan $\odot$, Volkan Kaynaroğlu๑ \\ Department of General Surgery, Hacettepe University School of Medicine, Ankara, Turkey
}

DOI: $10.18621 /$ eurj.424016

\begin{abstract}
Objectives: Idiopathic granulomatous mastitis (IGM) is an idiopathic, inflammatory, and benign disease of the breast. The aim of this study was to determine possible risk factors for the recurrence of IGM in patients treated with different treatment modalities.

Methods: It was planned to explore the risk factors of the disease through the evaluation of the medical records of 81 patients with the relevant diagnosis in the archives of Hacettepe University. The medical records of the patients were retrospectively screened for information regarding age, sex, total number of pregnancies, duration of breastfeeding, rheumatologic diseases, size and recurrence of the radiologically detected lesions. The results of two-year follow-up of the patients after the treatment were evaluated. The rates of recovery and recurrence after the treatment were determined for patients who received antibiotic therapy alone, who underwent wide excision alone, who received antibiotic therapy in combination with steroid therapy, and who received added azathioprine and/or methotrexate therapy.

Results: No significant difference was observed between 12 patients having recurrence and 69 patients having no recurrence. However, the recovery time was shorter for patients with a lesion size of $\leq 20 \mathrm{~mm}$ who underwent wide excision or received antibiotic therapy alone.

Conclusion: Among patients with a mass size $\leq 20 \mathrm{~mm}$, those treated with wide excision and antibiotic therapy alone had shorter recovery times compared with those who received immunosuppressive therapy. In addition, it may take more than two years to terminate the treatment in patients who received medical treatment alone.
\end{abstract}

Keywords: idiopathic granulomatous mastitis, recovery, recurrence, treatment, breast

Received: May 16, 2018; Accepted: November 14, 2018; Published Online: March 12, 2019

diopathic granulomatous mastitis (IGM) is a rare, chronic inflammatory disease of the breast with unknown etiology. IGM was first defined by Kessler and Wolloch [1] in 1972 as a non-malignant chronic inflammatory breast disease. The actual prevalence of IGM is unknown; however, histopathologically-confirmed IGM has been reported in $1.8 \%$ of 1106 patients with benign breast disease [2]. Galactorrhea, inflammation, induration, palpable mass and ulcera- tive lesions are observed in the IGM. The disease recurs in most patients and may be complicated with abscess and fistulas [3]. These complaints may easily be confused with breast cancer; however, IGM and breast cancer can be differentiated on the basis of clinical and radiological findings, although a tissue diagnosis is required for differentiation definitive diagnosis.

An autoimmune hypothesis is widely accepted in the pathophysiology of IGM. Protein-rich secretion

Address for correspondence: Hüseyin Onur Aydın, MD., Hacettepe University School of Medicine, Department of General Surgery, Ankara, Turkey E-mail: dronuraydin@hotmail.com,Tel:+90 312 2030520,Fax:+903122030521 
from ductal epithelial damage is thought to result in a granulomatous reaction stimulating the migration of lymphocytes and macrophages, first to the surrounding fat tissue, and then to the lobular connective tissue [4]. IGM is histopathologically-characterized by chronic granulomatous lobulitis without caseification [5].

The treatment of IGM remains controversial. Immunosuppressive therapy or surgical excision are preferable. Surgical treatment increases recurrence in complicated patients, and recovery may be achieved with close follow up in some patients [6]. In our hospital, patients diagnosed with IGM are treated with antibiotic therapy, immunosuppression, or surgical excision. In this study, we retrospectively investigated IGM patients to evaluate the efficacy of different treatment options. We compared the factors that possibly influenced the recurrence of IGM and patients' recovery times based on the chosen treatment method. Our aim was to determine possible risk factors for the recurrence of IGM in patients treated with different treatment modalities and increase the amount of available information for this unclear disease, by evaluating one of the largest known patient series.

\section{METHODS}

We retrospectively reviewed the records of patients who were diagnosed with granulomatous mastitis after being admitted to our clinic with redness, painful mass or purulent discharge.In this study, patients with other causes of granulomatous mastitis (sarcoidosis, tbc, etc.) were excluded. Eighty-nine patients histopathologically diagnosed with IGM between March 2003 and April 2012 were evaluated by retrospectively reviewing the archives of Hacettepe University School of Medicine and patient records. For diagnosis, excisional biyopsy was performed in 35 patients, incisional biyopsy in 22 patients and trucut biyopsy in 24 patients. Eight patients were excluded from the study because the details of their records could not be accessed from the system review. We evaluated the results of a two-year post-treatment patient's follow-up and recorded the rate of the response to treatment and the recurrence in patients who received antibiotic therapy alone, who underwent wide excision alone, who received antibiotic therapy in combination with steroid therapy, who received added azathioprine and methotrexate therapy in cases of resistance to initial treatment, or who underwent wide excision in cases of resistance to all initial treatments.

The recovered patients included those with symptom improvement and loss of mass size in twoyear follow-up. The recurrence was defined as the emergence of a recurrent mass or fistula during a twoyear follow-up of recovered patients. The persistent disease was defined as non-healing fistulas, abscess or mass during the treatment. Resection with surgical margin more than $1 \mathrm{~cm}$ was defined as wide excision. The choice of treatment method was made at the time of diagnosis, according to the current treatment recommendations in the literature. This study was approved by Hacettepe University Institutional Review Board (Project no: GO 13/230-29) and supported by Hacettepe University Research Fund.

Patients were treated with 40-60 $\mathrm{mg} /$ day prednisolone for 1 month. Then the prednisolone dosage was tapered to $30 \mathrm{mg}$ on alternate days over a period of 1-2 months, and after 6 months the dosage was $10-15 \mathrm{mg}$ on alternate days. The maintenance dosage of prednisolone was $5-7.5 \mathrm{mg}$ on alternate days. Steroid was stopped after 1.5-2 years. In the case of relapse, the dosage was increased to $40 \mathrm{mg}$ /day. Patients were treated with $2 \mathrm{mg} / \mathrm{kg}$ /day azathioprine as steroid-sparing agent as previously defined [7]. Methotrexate (MTX, 10-15 mg weekly, orally) was added in patients who had persistent disease and an unresolving breast mass despite steroid treatment. Also, MTX was introduced to help reduce prednisolone dosage, as previously reported [8]. All patients were re-examined within 3 months of therapy, and then at 6-month intervals.

The breast mass size was detected radiologically, using ultrasonography (USG), mammography (MMG) or magnetic resonance imaging (MRI) scans. Breast size was categorized into four groups: $\leq 10 \mathrm{~mm}, 11$ $20 \mathrm{~mm}, 21-30 \mathrm{~mm}$, and $\geq 31 \mathrm{~mm}$. The age at diagnosis, the duration of breast-feeding, abscess drainage, and the presence of fistula, erythema and ulceration in cases with recurrence, and the treatment outcomes were compared between these groups. We also evaluated recovery times on the effect of the treatment method. 


\section{Statistical Analysis}

The data of patients were analyzed using SPSS 21.0 software package (SPSS, Chicago, IL). The normality of distribution of study variables was assessed by Levene test of variance homogeneity by Shapiro-Wilk test. The quantitative variables were shown as median range (minimum- maximum), and the categorical variables were shown as numbers. To compare categorical variables, Chi-squared or Fisher's exact test was used. Mann-Whitney test was used to compare continuous variables between the groups, because the data were not normally distributed. Variables were examined at a confidence level of $95 \%$ and a significance level of $p<0.05$.

\section{RESULTS}

We evaluated 81 patients who were diagnosed histopathologically with IGM by examining the results of a mean of two-year post-treatment follow-up. All patients were female. The median age of the patients at diagnosis was 35 (range, 24-60) years. Patients had no history of diabetes mellitus and all had normal serum prolactin levels. Ten of the patients had no history of pregnancy. On other hand, the remaining patients had a history of pregnancy and labor. The median duration of breastfeeding was 10 (range, 6-24) months, and the maximum lesion size was $70 \mathrm{~mm}$. Unilateral disease was present in all of the patients and there was no difference in the incidence of breast side involvement. There was no correlation between recovery and recurrence rates and patients' age at diagnosis, duration of breastfeeding, and lesion size (Table 1). Recurrence was observed in 4 of 10 patients without a history of pregnancy and breastfeeding. One of four patients with recurrence underwent wide excision and the remaining three patients received prednisolone, azathioprine and methotrexate treatment. The shortest duration of treatment was one week in patients with wide excision, and 104 weeks in patients who received prednisolone, azathioprine, and methotrexate treatment.

There were no adverse events related to azathioprine such as cytopenia, liver disfunction, or severe infection. Four patient had temporary steroidinduced diabetes mellitus. In these patients, by reducing the dose of steroid glucose intolerance improved spontaneously and no additional treatment was needed. No steroid-related complications occurred in other patients.

Thirty-one patients underwent abscess drainage because of erythema, purulent discharge and subcutaneous fluid collection at admission and were

Table 1. Demographic and clinical parameters of recovery and recurrence groups

\begin{tabular}{lccc}
\hline Patients Characteristics & $\begin{array}{c}\text { Recovery } \\
(\mathbf{n = 6 9 )}\end{array}$ & $\begin{array}{c}\text { Recurrence } \\
(\mathbf{n = 1 2})\end{array}$ & $\boldsymbol{p}$ value \\
\hline Age (year) & $35(24-60)$ & $36(24-49)$ & 0.548 \\
Breast-feeding (month) & $12(6-24)$ & $12(6-12)$ & 0.122 \\
Number of child & $2(0-3)$ & $1(0-3)$ & 0.646 \\
Lesion size (mm) & $10(10-70)$ & $20(10-50)$ & 0.078 \\
Smoking & & & \\
Yes & 17 & 4 & 0.578 \\
No & 52 & 8 & \\
Lesion side & & & 0.236 \\
Right & 29 & 5 & \\
Left & 40 & 7 & 0.276 \\
Abscess drainage & & & \\
Yes & 25 & 6 & \\
No & 43 & 7 & \\
\hline Data give & & \\
\hline
\end{tabular}

Data are given as mean and ranges or number. 
Table 2. Comprasion of recovery and recurrent groups according to lesion size

\begin{tabular}{|c|c|c|c|}
\hline & $\begin{array}{c}\text { Recovery } \\
(n=69)\end{array}$ & $\begin{array}{l}\text { Recurrence } \\
(\mathbf{n}=12)\end{array}$ & $p$ value \\
\hline Lesion size (mm) (n) & & & 0.078 \\
\hline$\leq 10$ & 23 & 3 & \\
\hline $11-20$ & 22 & 2 & \\
\hline $21-30$ & 16 & 3 & \\
\hline$\geq 31$ & 6 & 4 & \\
\hline \multicolumn{4}{|l|}{ Treatment method (n) } \\
\hline None & 6 & 1 & \\
\hline Atb & 19 & 2 & \\
\hline $\mathrm{WE}+/-$ Atb & 14 & 2 & \\
\hline Pred +/- Mtx,Azat & 28 & 7 & \\
\hline Duration of treatment (median) (week) & & & 0.957 \\
\hline Atb & 4 & 6 & \\
\hline $\mathrm{WE}+/-\mathrm{Atb}$ & 1 & 3 & \\
\hline Pred +/- Mtx,Azat & 48 & 52 & \\
\hline
\end{tabular}

treated with oral antibiotics according to the culture results. Culture results revealed the growth of Bacteroides capillosus in one patient, Acinetobacter baumannii in one patient, Klebsiella in one patient and Staphylococcus epidermidis in four patients, with no resistant strains for ampicillin/sulbactam having been identified in the antibiogram results. Biopsy specimens from all patients were evaluated using acidalcohol-fast staining, and all were negative. Abscess drainage was performed in 31 patients; 25 patients recovered, and 6 patients had recurrence. Among these patients, 3 patients were treated with antibiotics, 1 patient was treated with antibiotic and wide excision, 1 patient was treated with prednisolone and azathioprine, and 1 patient was treated with prednisolone and methotrexate. In patients who had recovery, the shortest duration of treatment was 4 weeks and the longest duration of treatment was 52 weeks (antibiotic treatment and prednisolone with methotrexate treatment, respectively). There was no significant difference between the groups with and without abscess drainage with respect to recurrence $(p$ $=0.559$ ).

We excluded two patients from the lesion size group because of the lack of evidence to confirm the mass using USG and MMG in one patient, and because of the mass involving the entire breast in another. Eight patients refused treatment, in one of which there was a lack of evidence about the exact nature of the mass; however, all of them returned for outpatient evaluation at random intervals through the two-year follow-up. The median lesion size of these patients was $27 \mathrm{~mm}$ (range, 0-40 $\mathrm{mm}$ ) and, all of these patients had a history of breastfeeding. The median duration of breastfeeding was 12 (range, 6-12) months, and no significant difference was seen in this group. Seven patients in the "no treatment" group recovered, and one developed resistant fistulisation. After classifying the patients according to mass size, we compared the different treatment methods. Recurrence after follow-up without treatment or follow-up with antibiotics alone was lower in the $\leq 10 \mathrm{~mm}$ mass size group in comparison to the other groups. However, no significant difference was found between the groups. The effect of various treatment options on the time from the selected initial treatment to recovery was shown on Table 2. Two patients treated with prednisolone and methotrexate showed complete 
Table 3. Duration of treatment and recovery

\begin{tabular}{|c|c|c|c|c|}
\hline \multirow[b]{2}{*}{ Lesion size } & \multirow[t]{2}{*}{ Treatment method (n) } & \multicolumn{3}{|c|}{ Treatment time (week) } \\
\hline & & Mean & Minimum & Maximum \\
\hline \multirow{7}{*}{$\leq 10 \mathrm{~mm}$} & $\operatorname{ATB}(7)$ & 3.57 & 3.00 & 4.00 \\
\hline & Azat + Pred (6) & 60.67 & 52.00 & 104.00 \\
\hline & W.E. (4) & 26.75 & 1.00 & 104.00 \\
\hline & W.E. + ATB (3) & 3.33 & 3.00 & 4.00 \\
\hline & Pred (1) & 4.00 & 4.00 & 4.00 \\
\hline & Pred + MTX (3) & 104.00 & 52.00 & 156.00 \\
\hline & Pred + MTX + Azat (2) & 52.00 & 52.00 & 52.00 \\
\hline \multirow[t]{8}{*}{$11-20 \mathrm{~mm}$} & None (2) & 1.00 & 1.00 & 1.00 \\
\hline & ATB (8) & 3.50 & 3.00 & 4.00 \\
\hline & Azat + Pred (4) & 58.50 & 26.00 & 104.00 \\
\hline & W.E. (1) & 1.00 & 1.00 & 1.00 \\
\hline & W.E. + ATB (2) & 5.50 & 3.00 & 8.00 \\
\hline & Pred (2) & 27.50 & 3.00 & 52.00 \\
\hline & Pred + MTX (1) & 156.00 & 156.00 & 156.00 \\
\hline & Pred + MTX + Azat (4) & 65.00 & 52.00 & 104.00 \\
\hline \multirow[t]{6}{*}{ 21-30 mm } & None (3) & 3.33 & 1.00 & 8.00 \\
\hline & ATB (4) & 4.25 & 1.00 & 8.00 \\
\hline & Azat + Pred (6) & 60.67 & 26.00 & 104.00 \\
\hline & W.E. (2) & 1.00 & 1.00 & 1.00 \\
\hline & W.E. + ATB (1) & 4.00 & 4.00 & 4.00 \\
\hline & Pred + MTX + Azat (3) & 69.33 & 52.00 & 104.00 \\
\hline \multirow[t]{5}{*}{$\geq 31$} & None (2) & 1.00 & 1.00 & 1.00 \\
\hline & ATB (2) & 4.00 & 4.00 & 4.00 \\
\hline & Azat + Pred (2) & 52.00 & 52.00 & 52.00 \\
\hline & W.E. (3) & 1.33 & 1.00 & 2.00 \\
\hline & Pred + MTX + Azat (1) & 52.00 & 52.00 & 52.00 \\
\hline
\end{tabular}

$\mathrm{Atb}=$ antibiotics, $\mathrm{WE}=$ wide excision, Pred $=$ prednisolone, $\mathrm{Mtx}=$ methotrexhate, Azat $=$ azathioprine, $\mathrm{n}=$ number of patients, $p=0.370$.

recovery after approximately 3 years. One patient treated with wide excision showed complete recovery after 2 years. We found no significant difference between the treatment methods with respect to recurrence independent of mass size. The recovery time was shorter both after antibiotic therapy and after wide excision in cases with the mass size $\leq 20 \mathrm{~mm}$. There was no statistical difference between differing lesion sizes with respect to the effects of the treatment methods on the duration of treatment, $(p=0.370)$
(Table 3). There were 9 patients who had mass size of $\leq 20 \mathrm{~mm}$ and treated with wide excision and antibiotic therapy, among whom only 1 patient had recurrent fistulas. At the end of the treatments all of patients had a complete recovery.

\section{DISCUSSION}

The two defined GM types are specific and 
idiopathic types [9]. Specific GMs are seen in diseases causing granulomas, such as tuberculosis, sarcoidosis, mycotic and parasitic infections. IGM is characterized by non-caseified granulomatous inflammation, necrosis, giant cell formation, and neutrophil migration. Granulomatous changes are observed around the lobules and ducts in the breast in the absence of other diseases such as infection, trauma, foreign body reaction, or tuberculosis [10]. IGM is a benign disease; however, it may be confused with the local findings of breast cancer at admission.

IGM usually occurs in young women under the age of 50 years with a history of labor and lactation $[10,11]$. There are only two cases of male patients from 537 patients published in the literature between 1972 and $2010[12,13]$. All patients evaluated in the present study were female, and $87.5 \%$ of these patients had a history of pregnancy and lactation.

There was no significant relationship with duration of breast-feeding, total number of pregnancies and oral contraceptive use [14]. Former reports suggested that prolonged duration of breastfeeding could lead to distention of acini and ducts in the long-term, resulting in rupture, which might induce a granulomatous response [15]. This theory is partially true, but unexplained contralateral breast involvement alone has occurred in patients breastfeeding from only the opposite breast. Ten patients had no history of pregnancy in our evaluation. The remaining 71 patients had a history of pregnancy and labor. The median duration of breastfeeding was 10 months. The number of pregnancies and the duration of breastfeeding did not have a significant effect on recovery or recurrence.

Different rates of bilateral disease have been reported in the literature, with no significant difference being present between left and right breast involvement $[16,17]$. In our study, three patients had bilateral involvement; 31 patients had right breast involvement; and 47 patients had left breast involvement, with no significant difference between both sides. Based on interviews with patients, they preferred bilateral breast-feeding.

Previous publications have reported a history of prolonged treatment duration in the presence of Group D Corynebacterium infection [18]. Thirty-one of our patients underwent abscess drainage because of purulent discharge, erythema and fluctuation at admission. The growth of Staphylococcus and its strains were observed only in ten patients. Corynebacterium was not isolated from these patients; however, as mentioned by Mathelin et al., [18] Corynebacterium may be found in repeat cultures in treatment-resistant cases; therefore, once it is isolated, treatment response may be evaluated on the basis of antibiotic therapy.

Lai et al. [19] investigated nine patients with IGM. Lumpectomy was performed only in one patient because of large lesion size, with no recurrence during the 30-month follow-up in this patient. The remaining eight patients received no surgical intervention or medical treatment, and complete recovery was observed in four patients during the 24-month followup. The disease remained stable during the 11-month follow-up after the first 12-month recovery period in four patients, and no abscess formation or fistulization was seen during the follow-up of any patient. These findings suggest that the disease has a self-limiting nature and complete recovery is achievable during conservative follow-ups. The authors also recommended using high-dose steroid therapy in recurrent cases with multiple fistulas. In our study, seven patients received no medical or surgical treatment, and only one patient had resistant fistulization, which was treated by wide excision and antibiotics.

A study by Konan et al. [20] from Hacettepe University Hospital investigated 15 patients diagnosed with IGM. Steroid therapy alone was administered to one of these patients because of concurrent pregnancy. The remaining 14 patients received simultaneous prednisolone and azathioprine. Prednisolone was maintained for 12-24 months with tapering the dose. No patients experienced pancytopenia or impaired liver function secondary to azathioprine during followup; however, four patients developed temporary steroid-related diabetes mellitus. Complete recovery was observed in 11 patients during the 6-month follow-up. Recurrence was observed in two patients at the $4^{\text {th }}$ and $12^{\text {th }}$ months, with a partial response in one patient. Kim et al. [8] reported the use of methotrexate with steroids in four patients with IGM]. During the medical therapy none of their patients had recurrence, after cessation of methotrexate treatment, 
they reported two recurrences. Also, Raj et al. [7] reported three cases treated with medical therapy]. In their cases one patient had shortness of breath on methotrexate, which wasswitched to azathioprine treatment. All of three patients had complete recovery with medical therapy. Methotrexate is commonly used in autoimmune diseases such as rheumatoid arthritis. A low dose can help reduce concomitant steroid dose, while at the same time controlling the underlying autoimmune conditions. Also, azathioprine is widely used as a steroid-sparing agent after the initial control of autoimmune disease. It is a purine antagonist and inhibits both cellular and humoral immunity. Our data showed that 37 patients received medical treatment, with seven experiencing recurrent disease.

Surgical treatment of IGM is controversial because of delayed wound healing, high rates of local recurrence, and poor cosmetic outcomes. Yau et al. [21] carried out a retrospective analysis of 11 patients diagnosed with IGM treated at a tertiary care center. Eight patients had multiple surgical interventions because of recurrence. Five patients had excisional biopsy; five patients had partial mastectomy; and one patient had total mastectomy and transverse rectus abdominis musculocutaneous flap. Recurrence was identified in one patient who had undergone partial mastectomy. Most authors suggested that definitive surgical interventions exacerbate the clinical course because of local recurrence and poor cosmetic outcomes. Moreover, Yabanoglu et al. [22] reported successful surgical outcomes in patients with IGM. They compared 31 wide excision, one simple mastectomy and one subcutaneous mastectomy results to 44 steroid treatment results. In the surgical treatment group, they reported no recurrences, whereas in the medical treatment group nine patients had recurrence. In our study, the recovery time was shorter with wide excision and antibiotic therapy alone when necessary compared with immunosuppressive therapy in cases with the mass size $\leq 20 \mathrm{~mm}$.

\section{Limitations}

The retrospective observational nature of our study was its limitation. It was difficult to assess a disease which pathophysiology was not fully understood. It is also difficult to compare the different treatment modalities at different time points. Nonetheless, it was important by being one of the largest series in the literature. Large patient populations and different treatment regimens were evaluated in a single study.

\section{CONCLUSION}

There is a limited number of reported cases of IGM in the literature because the disease is so rare. In this study, the size of the patient series used was quite extensive, that is 81 patients. No significant difference was found between the efficacy of different treatment methods administered according to patients' characteristics and the effect on recurrence. However, among cases with the mass size $\leq 20 \mathrm{~mm}$, those treated with wide excision and antibiotic therapy alone had shorter recovery times compared with the patients who received immunosuppressive therapy. In addition, it may take more than two years to terminate treatment in patients treated with medical treatment alone.

We determined that no factor significantly affected the recovery and recurrence rates. Future multicenter prospective randomized controlled trials employing standardized treatment methods will help to establish factors affectingrecovery and recurrence rates in the disease.

\section{Conflict of interest}

The authors disclosed no conflict of interest during the preparation or publication of this manuscript.

\section{Financing}

The authors disclosed that they did not receive any grant during conduction or writing of this study.

\section{REFERENCES}

[1] Kessler E, Wolloch Y. Granulomatous mastitis: a lesion clinically simulating carcinoma. Am J Clin Pathol 1972;58:6426.

[2] Baslaim MM, Khayat HA, Al-Amoudi SA. Idiopathic granulomatous mastitis: A heterogeneous disease with variable clinical presentation. World J Surg 2007;31:1677-81.

[3] Ozel L, Unal A, Unal E, Kara M, Erdoğdu E, Krand O, et al. Granulomatous mastitis: is it an autoimmune disease? Diagnostic and therapeutic dilemmas. Surg Today 2012;42:729-33.

[4] Taylor GB, Paviour SD, Musaad S, Jones WO, Holland DJ. A clinicopathological review of 34 cases of inflammatory breast disease showing an association between corynebacteria infection 
and granulomatous mastitis. Pathology 2003;35:109-19.

[5] Akcan A, Akyildiz H, Deneme MA, Akgun H, Aritas Y. Granulomatous lobular mastitis: A complex diagnostic and therapeutic problem. World J Surg 2006;30:1403-9.

[6] Hugon-Rodin J, Plu-Bureau G, Hugol D, Gompel A. Management of granulomatous mastitis: a series of 14 patients. Gynecol Endocrinol 2012;28:921-4.

[7] Raj N, Macmillan RD, Ellis IO, Deighton CM. Rheumatologists and breasts: immunosuppressive therapy for granulomatous mastitis. Rheumatology 2004;43:1055-6.

[8] Kim j, Tymms KE, Buckingham JM. Methotrexate in the management of granulomatous mastitis. ANZ J Surg 2003;73:247-9.

[9] Ocal K, Dag A, Turkmenoglu O, Kara T, Seyit H, Konca K. Granulomatous mastitis: clinical, pathological features, and management. Breast J 2010;6:176-82.

[10] Tavassoli FA. Idiopathic granulomatous mastitis. In: Tavassoli FA, eds. Pathology of the Breast. 2nd ed. New York, NY: McGraw-Hill. 1999:793-794.

[11] Erhan Y, Veral A, Kara E, Ozdemir N, Kapkac M, Ozdedeli E, et al. A clinicopathologic study of a rare clinical entity mimicking breast carcinoma: idiopathic granulomatous mastitis. Breast 2000;9:52-56.

[12] Reddy KM, Meyer CE, Nakdjevani A, Shrotria S. Idiopathic granulomatous mastitis in the male breast. Breast J 2005;11:73.

[13] Tse GM, Poon CS, Ramachandram K, Ma TK, Pang LM, Law BK, et al. Granulomatous mastitis: a clinicopathological review of 26 cases. Pathology 2004;36:254-7.
[14] Gal-Gombos EC, Esserman LE, Weisberg S. Granulomatous mastitis. J Womens Imaging 2004;6:136-9.

[15] Kaur AC, Dal H, Muezzinoglu B, Paksoy N. Idiopathic granulomatous mastitis. Report of a case diagnosed with fine needle aspiration cytology. Acta Cytol 1999;43:481-4.

[16] DeHertogh DA, Rossof AH, Harris AA, Economou SG. Prednisone management of granulomatous mastitis. N Engl J Med 1980;303:799-800.

[17] Patel RA, Strickland P, Sankara IR, Pinkston G, Many W Jr, Rodriguez M. Idiopathic granulomatous mastitis: case reports and review of literature. J Gen Intern Med 2010;25:270-3.

[18] Mathelin C, Riegel P, Chenard MP, Tomasetto C, Brettes JP. Granulomatous mastitis and corynebacteria: clinical and pathologic correlations. Breast J 2005;11:357.

[19] Lai EC, Chan WC, Ma TK, Tang AP, Poon CS, Leong HT. The role of conservative treatment in idiopathic granulomatous mastitis. Breast J 2005;11:454-6.

[20] Konan A, Kalyoncu U, Doğan Ü, Kılıç YA, Karakoç D, Akdogan A, et al. Combined long-term steroid and immunosuppressive treatment regimen in granulomatous mastitis. Breast Care 2012;7:297-301.

[21] Yau FM, Macadam SA, Kuusk U, Nimmo M, Van Laeken $\mathrm{N}$. The surgical management of granulomatous mastitis. Ann Plast Surg 2010;64:9-16.

[22]Yabanoğlu H, Çolakoğlu T, Belli S, Ataç HO, Bolat FA, Pourbagher A, et al. A comparative study of conservative versus surgical treatment protocols for 77 patients with idiopathic granulomatous mastitis. Breast J 2015;21:363-9. 